\title{
Potential of Hyper-Kamiokande at some non-Accelerator Physics and Nucleon Decay Search Programs
}

\section{Luis Labarga ${ }^{* t}$}

University Autonoma Madrid

E-mail: luis. labarga@uam.es

on behalf of The Hyper-Kamiokande proto-Collaboration

\begin{abstract}
Hyper-Kamiokande is a next generation underground water Cherenkov detector based on the highly successful Super-Kamiokande experiment. It will be capable of observing - far beyond the sensitivity of the Super-Kamiokande detector - proton decay, atmospheric neutrinos, and neutrinos from astronomical sources. It will also serve as a far detector, $295 \mathrm{~km}$ away, of a long baseline neutrino experiment for the upgraded J-PARC beam.

Further to the detector planned size of more than one order of magnitude larger than predecessor experiments, its improved photon yield will enable superior signal efficiency and background rejection. This presentation will describe the physics potential of Hyper-Kamiokande's for some searches of Dark Mater and, mostly, for nucleon decay. This will be illustrated with the relevant decay modes $p \rightarrow e^{+} \pi^{0}$ and $p \rightarrow v K^{+}$.
\end{abstract}

38th International Conference on High Energy Physics

3-10 August 2016

Chicago, USA

\footnotetext{
${ }^{*}$ Speaker.

${ }^{\dagger}$ supported by EU H2020-MSCA-RISE-2014-GA641540, SKPLUS.
} 
On the strength of a double Nobel prize winning experiment (Super)Kamiokande and an extremely successful long baseline neutrino programe, the third generation Water Cherenkov detector, Hyper-Kamiokande, is being developed by an international collaboration as a leading worldwide experiment based in Japan [1]. It will address the biggest unsolved questions in physics through a multi-decade physics programe that will start in the middle of the next decade.

The Hyper-Kamiokande detector will be hosted in the Tochibora mine (Kamiola, Gifu Prefecture, Japan). The currently existing accelerator in J-PARC will be steadily upgraded to reach a MW beam by the start of the experiment. Figure 1 shows a global picture, with relevant details, of the experiment as a whole. The experiment will be the largest underground water Cherenkov detector in the world and will be instrumented with new technology photosensors (PMTs), faster and with higher quantum efficiency than the ones in Super-Kamiokande [2] (see Fig. 2).

Hyper-K is a truly international proto-collaboration with over 60 participating institutions from Brazil, Canada, the United States, France, the United Kingdom, Italy, Korea, Poland, Russia, Spain, and Switzerland, in addition to Japan. Hyper-K will be a multipurpose neutrino detector with a rich physics program that aims to address some of the most significant questions facing particle physicists today. Oscillation studies from accelerator [2], atmospheric and solar neutrinos [2] will refine the neutrino mixing angles and mass squared difference parameters and will aim to measure a CP-violating phase in the leptonic sector that will be pivotal in the understanding of the matterantimatter asymmetry in the Universe. The search for nucleon decays will probe one of the key tenets of Grand Unified Theories. In the case of a nearby supernova, Hyper-K will observe an unprecedented number of neutrino events, providing the much needed experimental results to understand the mechanism of the explosion [2]. The detection of astrophysical neutrinos from sources such as dark matter annihilation will revolutionize our knowledge of one of the least understood phenomena in the Universe.

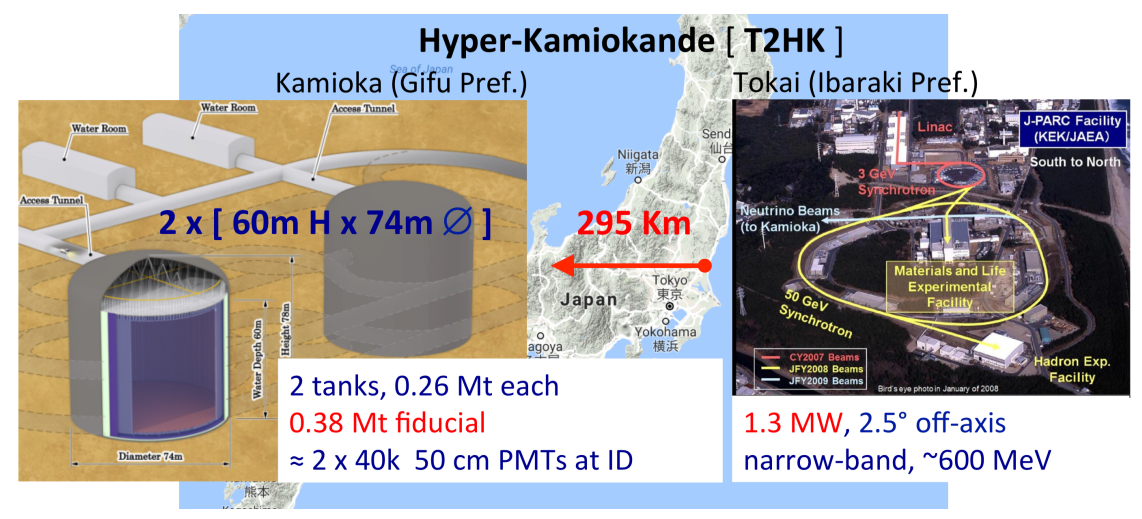

Figure 1: The Hyper-Kamiokande project and T2HK long base line experiment.

\section{Photosensors}

The Hyper-K's inner detector (ID) photosensor, Hamamatsu R12860-HQE, was newly developed based on the well established and reliable design of Super-K's 50cm R3600 PMT by Hamamatsu Photonics K.K. and KamLAND's Hamamatsu R7250. It features a significantly faster time response, better charge resolution and a higher detection efficiency with a stable mechanical structure, compared to the existing large aperture PMTs. These properties have a particularly relevant 
impact on the achievable solar- $v$ physics, neutron tagging by proton capture and the reconstruction of the proton-decay mode $p \rightarrow \bar{v} K^{+}$.

The Quantum Efficiency $(\mathrm{QE})$ reaches $\sim 31 \%$ at $\approx 400 \mathrm{~nm} ; 41 \%$ larger than the conventional R3600 (see Fig. 2 top-left). Figure 2 top-right shows the detection efficiency, twice as high as R3600's, and its good uniformity over the whole PMT surface in spite of the asymmetric dynode structure. The single photoelectron pulse in a HQE B\&L PMT has a $6.7 \mathrm{~ns}$ rise time (10\%-90\%) and 13.0ns FWHM without ringing, which is faster than the 10.6ns rise time and 18.5ns FWHM in the Super-K PMT. The time resolution for single photo-electrons (PEs) is $1.1 \mathrm{~ns}$ in $\sigma$ for the fast left side of the transit time peak (Fig. 2 bottom-right) and 4.1ns at FWHM. This impacts very positively the reconstruction performance of events in Hyper-K. The nominal gain is 107 and can be adjusted for several factors in a range between $1500 \mathrm{~V}$ to $2200 \mathrm{~V}$. Fig. 2 bottom-left shows the charge distribution, where the $35 \%$ resolution in $\sigma$ of the single PE is to be compared to the $50 \%$ of the Super-K PMT. The peak-to-valley ratio is about 4.
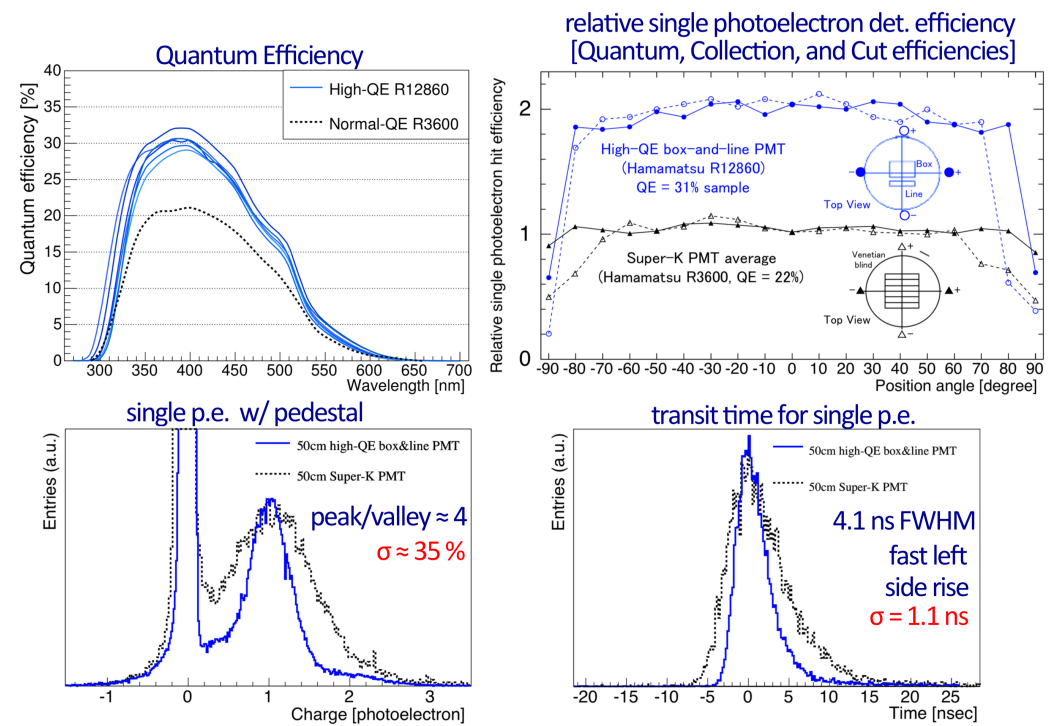

Figure 2: top-left: Measured QE for six high-QE R12860 (solid lines) and a normal R3600 (dashed line). Top-right: Relative single photoelectron detection efficiency as a function of the position in the photocathode. The dashed line is the scan along the symmetric line of the box-and-line dynode whereas the solid line is along the perpendicular direction of the symmetric line. The detection efficiency represents QE, CE and cut efficiency of the single photoelectron at 0.25 PE. Bottom-left: Single photoelectron distribution with pedestal. Bottom-right: Transit time distribution at single photoelectron.

\section{Searches for Dark Matter}

The self-interaction or decay of dark matter particles bound in strong gravitational potentials, such as those of the milky way galaxy or our sun, may produce standard model particles. Either through direct annihilation (decay) into neutrinos or through the decays of heavier particles that include $v$ final states, it is possible to observe these $v$ secondaries at Hyper-Kamiokande. In this case the atmospheric $v$ sample becomes an overwhelming background. However, a potential dark matter signal is expected to have an angular distribution peaked sharply near the center of the binding potential, which at Hyper-Kamiokande would manifest as the direction towards the galactic center or the sun, whereas the atmospheric neutrino background is expected to be uniformly distributed 
in this coordinate. The momentum distribution of the signal is used for extracting the mass of the dark matter candidate producing any observed neutrino event excess.

From the quantitative comparison between data distributions and the expected atmospheric backgrounds, limits on the WIMP-induced neutrino flux are translated into limits on the WIMPnucleon spin-dependent (SD) and spin-independent (SI) cross sections using the DarkSUSY simulation. The sensitivity of Hyper-K to solar WIMPS is shown in Fig. 3 (for limits on galactic-center WIMPS see [1]). The left (right) plot is for the WIMP-proton SD (WIMP-nucleon SI). Also shown are cross section (hatched areas) and limits (shown as lines) from current experiments. Hyper-K is expected to produce limits a factor of $3 \sim 4$ times Super-K's (black curves). Further, current hints for a positive SI (SD) dark matter signal [4], can be probed completely by Hyper-K's $\tau^{+} \tau^{-}$channel (all channels). For SI interactions Hyper-K will provide sensitivity to sub $10 \mathrm{GeV} / c^{2}$ masses, a region of difficult access to direct detection experiments using current technologies.
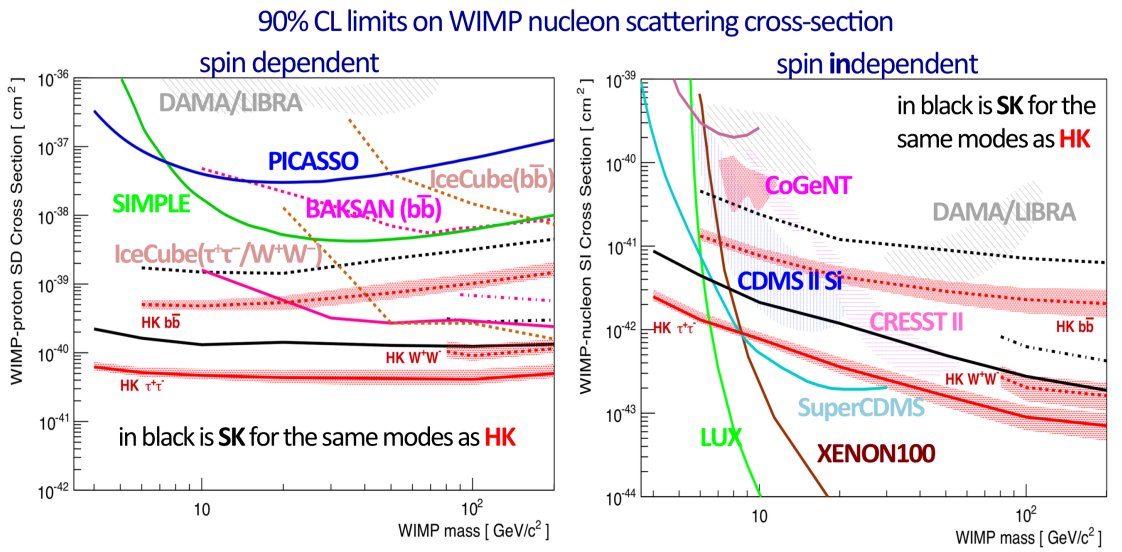

Figure 3: The 90\% C.L. upper limits on the SD (left) and SI (right) WIMP-nucleon scattering cross section based on a search from WIMP-induced neutrinos coming from the direction of the sun are shown as red curves as function of WIMP mass. Limits (lines) and allowed regions (hatched areas) from other experiments are also shown. Results from Super-Kamiokande appear in black [3]. Bands around the Hyper-K limits indicate the combined effect of uncertainties in the solar model, nuclear form factor, and assumed WIMP velocity distribution.

\section{Grand Unification}

A primary goal of Hyper-Kamiokande is the thorough experimental study of Grand Unification by the search and maybe observation and discovery of a nucleon decay signal. One of the strengths of the water Cherenkov technology is its sensitivity to a wide variety of decay modes. Using Monte Carlo and analysis techniques originally developed for Super-Kamiokande, we show here Hyper$\mathrm{K}$ 's expected sensitivity to the two flagship proton decay modes, $p \rightarrow \bar{v} K^{+}$and $p \rightarrow e^{+} \pi^{0}$. For expectations for other $\Delta(\mathrm{B}-\mathrm{L})$ conserving, $\Delta \mathrm{B}=2$ dinucleon, and $\Delta(\mathrm{B}-\mathrm{L})=2$ decays, see [1].

Proton decays into a lepton and a kaon are a feature of supersymmetric grand unified theories. Searching for the $K^{+}$in a water Cherenkov detector is complicated by the fact that it emerges from the decay with a momentum of only $340 \mathrm{MeV} / \mathrm{c}$, well below the threshold for light production of $749 \mathrm{MeV} / \mathrm{c}$. As a result the $K^{+}$must be identified by its decay products: $K^{+} \rightarrow \mu^{+} v(64 \%$ branching fraction) and $K^{+} \rightarrow \pi^{+} \pi^{0}$ (21\% branching fraction). Since both of these modes are two body decays the outgoing particles have monochromatic momenta. Furthermore, the 12 ns lifetime 
of the kaon makes it possible to observe prompt $\gamma$-ray emission produced when the proton hole leftover from a bound proton decay is filled by the de-excitation of another proton. For ${ }^{16} \mathrm{O}$ nuclei the probability of producing a $6 \mathrm{MeV} \gamma$ from such a hole is roughly $40 \%$, making this a powerful tool for identifying the $K^{+}$decay products and rejecting atmospheric neutrino backgrounds. The reconstruction of those features by Hyper-K are illustrated in Fig. 4.
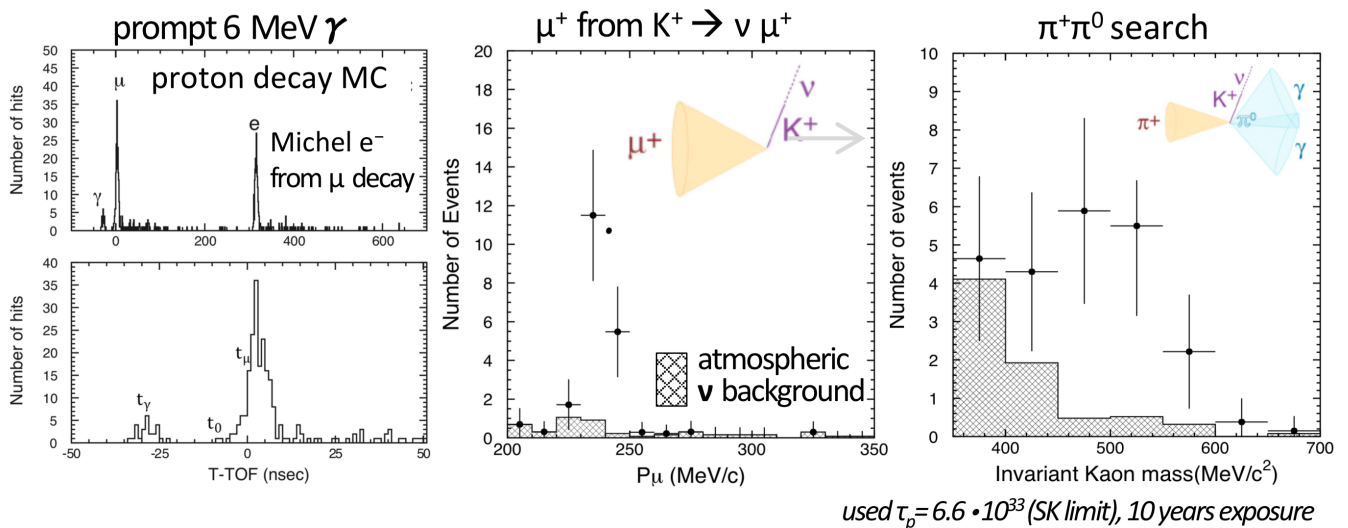

Figure 4: Left: Schematic view of the expected timing distribution of events in the prompt $\gamma$ search method for $p \rightarrow \bar{v} K^{+}$decays. Top: full event time window. Bottom: time of flight subtracted timing distribution around the $\mu$ candidate. Center: Reconstructed $\mu$ momentum distributions for muons found in the prompt $\gamma$ search. The solid crosses denote the sum of the background and proton decay signal. Right: Reconstructed kaon mass based on the reconstructed final state $\pi^{+} \pi^{0}$. These expectations are for the 1TankHD design after a 10 year run with a second tank coming online six years after the start of the experiment.

Three methods, each targeting different aspects of the $K^{+}$decay, are used to search for $p \rightarrow$ $\bar{v} K^{+}$events (see [5] for the details of the analyses). Figure 5 shows the $3 \sigma p \rightarrow \bar{v} K^{+}$discovery potential (left) and the sensitivity to this mode mode at $90 \%$ C.L. in case of no signal (right), as a function of run time as well as those of other planned experiments.

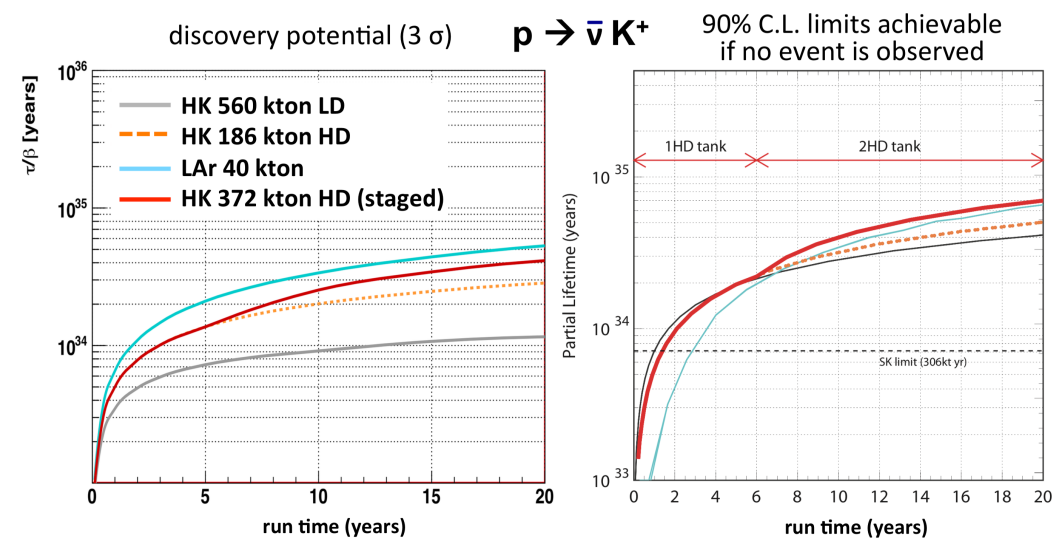

Figure 5: Left: Comparison of the $3 \sigma p \rightarrow \bar{v} K^{+}$discovery potential as a function of run time of the Hyper-K 1TankHD (red solid) and 3TankLD (gray solid) designs as for a 40 kton liquid argon detector (cyan solid). In the 1TankHD line an additional tank is assumed to come online six years after the start of the experiment and a dashed orange line denotes the potential of just a single tank. The 40 kton liquid argon detector is assumed to have a signal efficiency of $45 \%$ and an expected background of 1.0 events per Megaton·year. Systematic errors are (are not) included for Hyper-K (the LAr detector). Right: Assuming no proton decay signal, Hyper-K's sensitivity to $p \rightarrow \bar{v} K^{+}$at $90 \%$ C.L. as a function of run time for the same configurations. 
Proton decay into a positron and neutral pion is a favored mode of many GUT models. Experimentally this decay has a very clean event topology, with no invisible particles in the final state, being possible to fully reconstruct the proton's mass from its decay products. As it is a two body process the total momentum of the recoiling system should be small. Figure 6-left shows the expected distribution of this variable for both signal events and atmospheric $v$ backgrounds. Figure 6-middle shows the $3 \sigma p \rightarrow e^{+} \pi^{0}$ discovery potential and, (right) the sensitivity to this mode at $90 \%$ C.L. in case of no signal, as a function of run time, as well as those for other planned experiments.
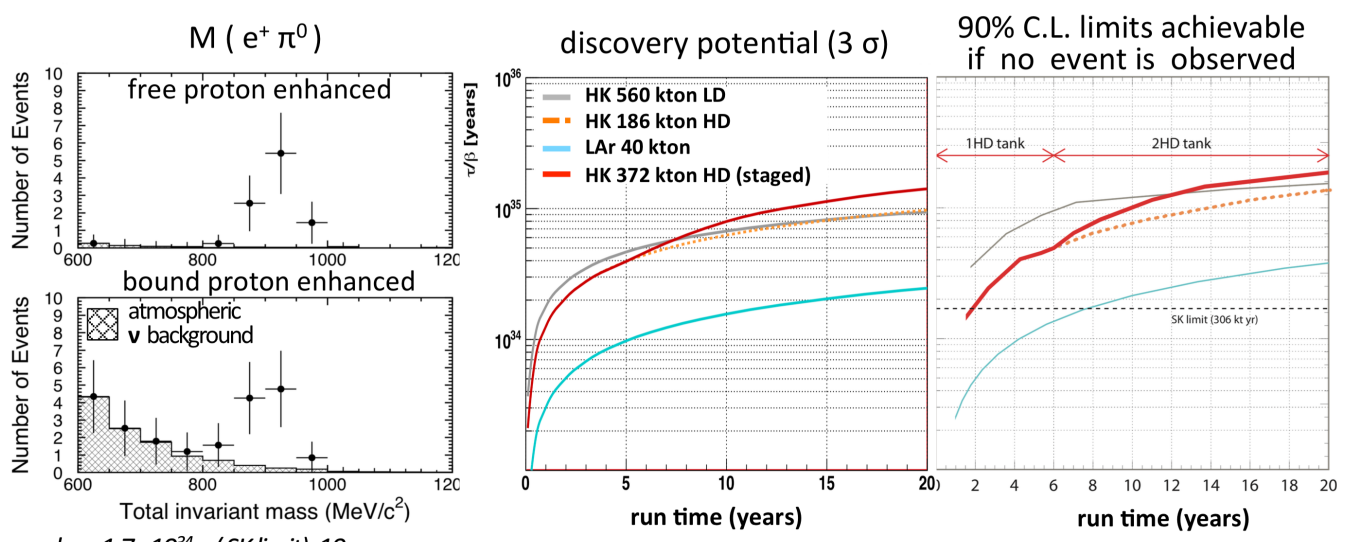

used $\tau_{p}=1.7 \cdot 10^{34} y$ (SK limit), $10 y$. exposure

Figure 6: Left: Reconstructed invariant mass distribution of events passing all steps of the $p \rightarrow e^{+} \pi^{0}$ event selection except the invariant mass cut. The solid crosses denote the sum of the atmospheric background and proton decay signal. The expectation is for the 1TankHD design with one additional tank is coming online six years after the start of the experiment. Middle: The $3 \sigma p \rightarrow e^{+} \pi^{0}$ discovery potential as a function of run time for the same configurations as in Fig. 5. Right: Assuming no proton decay signal, Hyper-K's sensitivity to the $p \rightarrow e^{+} \pi^{0}$ decay mode at $90 \%$ C.L. as a function of run time for the same configurations as in Fig. 5.

Epilogue. Hyper-Kamiokande is the next generation Megaton-like, neutrino and nucleon decay experiment. We have presented some of its superb physics expectations. Remarkably, HyperKamiokande will provide by far the most complete experimental exploration of Grand Unification within the next decades.

\section{References}

[1] Hyper-Kamiokande proto-Collab.; K. Abe et al., KEK Preprint 2016-21, ICRR-Report 701-2016-1

[2] Y. Nishimura, New $50 \mathrm{~cm}$ Photo-Detectors for HK; Talk at this Conference; Contribution \#638 M. Gonin, HK's Neutrino Oscillation Physics Sensitivity; Talk at this Conference; Contribution \#1038 L. Labarga, Astrophysics Potential of HK; Poster at this Conference; Contribution \#635

[3] Super-Kamiokande Collab.; K. Choi et al., Phys. Rev. Lett. 114, 141301 (2015)

[4] R. Bernabei et al. (DAMA), Eur. Phys. J. C56, 333 (2008)

C. Savage et al., JCAP 0909, 036 (2009)

R. Agnese et al. (CDMS), Phys. Rev. Lett. 111, 251301 (2013)

G. Angloher et al., Eur. Phys. J. C72, 1971 (2012)

C. E. Aalseth et al. (CoGeNT), Phys. Rev. Lett. 106, 131301 (2011)

[5] Super-Kamiokande Collab.; K. Abe et al., Phys. Rev. D90, 072005 (2014) 